\title{
Review of technological advancements in calibration systems for laser vision correction
}

Samuel Arba-Mosquera

Paolo Vinciguerra

Shwetabh Verma 


\title{
Review of technological advancements in calibration systems for laser vision correction
}

\author{
Samuel Arba-Mosquera, ${ }^{\mathrm{a}, \star}$ Paolo Vinciguerra, ${ }^{\mathrm{b}}$ and Shwetabh Verma ${ }^{\mathrm{a}, \mathrm{c}, \mathrm{d}, \mathrm{e}}$ \\ aSCHWIND Eye-Tech-Solutions, Research and Development, Kleinostheim, Germany \\ bIstituto Clinico Humanitas, Department of Ophthalmology, Milan, Italy \\ 'Heidelberg University, University Medical Center Mannheim, Experimental Radiation Oncology, Heidelberg, Germany \\ ${ }^{d}$ Heidelberg University, Interdisciplinary Center for Scientific Computing (IWR), Heidelberg, Germany \\ ${ }^{\text {eH}}$ Heidelberg University, Central Institute for Computer Engineering (ZITI), Heidelberg, Germany
}

\begin{abstract}
Using PubMed and our internal database, we extensively reviewed the literature on the technological advancements in calibration systems, with a motive to present an account of the development history, and latest developments in calibration systems used in refractive surgery laser systems. As a second motive, we explored the clinical impact of the error introduced due to the roughness in ablation and its corresponding effect on system calibration. The inclusion criterion for this review was strict relevance to the clinical questions under research. The existing calibration methods, including various plastic models, are highly affected by various factors involved in refractive surgery, such as temperature, airflow, and hydration. Surface roughness plays an important role in accurate measurement of ablation performance on calibration materials. The ratio of ablation efficiency between the human cornea and calibration material is very critical and highly dependent on the laser beam characteristics and test conditions. Objective evaluation of the calibration data and corresponding adjustment of the laser systems at regular intervals are essential for the continuing success and further improvements in outcomes of laser vision correction procedures. ๑ 2018 Society of Photo-Optical Instrumentation Engineers (SPIE) [DOI: 10.1117/1.JBO.23.2.020901]
\end{abstract}

Keywords: calibration systems; calibration materials; calibration methods; laser vision correction; laser beam characteristics; surface roughness.

Paper 170554VRR received Aug. 21, 2017; accepted for publication Jan. 10, 2018; published online Feb. 6, 2018.

\section{Introduction}

Laser vision correction procedures have seen several advancements in past decades, either due to the improvements in the clinical techniques being utilized or due to the technical advancements in the laser systems. Modern laser systems are much more sophisticated and offer submicron precision to successfully treat higher order aberrations by etching complex shapes on the cornea. However, it is imperative to calibrate and maintain these systems to repeatedly deliver the same standards of performance over their entire life cycle. This also makes the development of equally precise calibration systems a challenging task for the laser device manufacturers. Figure 1 shows an overview of the cycle of calibration normally followed in laser systems for laser vision correction.

A laser system sends regular feedback to the calibration device, which measures the deviation with respect to the expected signal. This regular feedback can be laser pulses directed on to the calibration device (for example, in case of energy measurement) at regular intervals or simply a prompt to use the calibration device and perform a system calibration (for example, in case of plastic ablations requiring user interaction). The deviation of the achieved signal from the expected signal acts as calibration data, which is interpreted either objectively or subjectively. The resulting adjustment factor is fed to the laser system for calibrating the system performance closer to the expectation. This cycle is repeated at different frequencies

*Address all correspondence to: Samuel Arba-Mosquera, E-mail: samuel.arba. mosquera@eye-tech.net depending on the frequency of the regular feedback, with different methods. With several calibration materials, methods, and devices either commercially available or under research and development, there is a need to review the accuracy introduced by each of these aspects and to find a configuration that reflects an optimum to achieve better outcomes after laser vision correction.

\section{Calibration Materials}

\subsection{Polymethylmethacrylate}

Excimer laser photoablation for refractive and therapeutic keratectomies has been demonstrated to be feasible and practicable. However, corneal laser ablations are not without problems, including the delivery and maintenance of a homogeneous beam. Different excimer laser calibration systems capable of characterizing a laser ablation profile have been developed. ${ }^{1}$ Beam homogeneity can be determined by the analysis of a polymethylmethacrylate (PMMA)-based thin film using video capture and image processing. Analysis and interpolation of excimer calibration system data can provide a three-dimensional (3-D) representation of elevation profiles, which correlates with two-dimensional scanning profilometry. Detection of excimer laser beam ablation profiles may be useful for precise calibration of excimer lasers before human photorefractive and therapeutic surgery. This was demonstrated in a study with excimer calibration analysis using PMMA, performed before treating a monkey undergoing phototherapeutic keratectomy (PTK) and two human subjects undergoing myopic spherocylindrical photorefractive keratectomy. ${ }^{1}$ Excimer calibration analysis predicted 


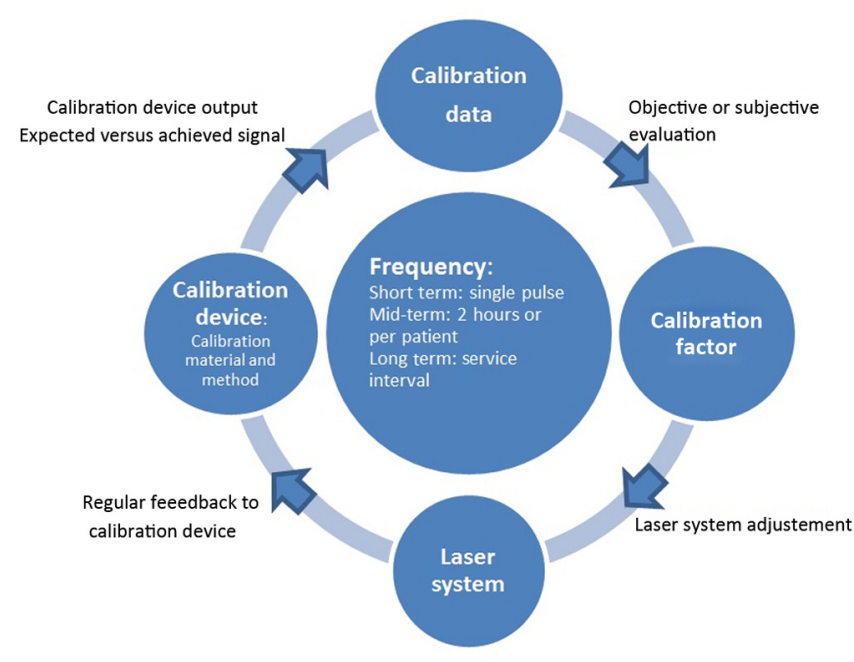

Fig. 1 Cycle of calibration followed in laser systems for laser vision correction.

a central-steep-island ablative pattern that was confirmed by corneal topography immediately postoperatively and at 1 week after re-epithelialization both in the monkey and human subjects. Subsequent technical refurbishing of the laser resulted in a beam with an overall increased ablation rate measured as microns/pulse with a donut ablation profile. A patient treated after repair of the laser electrodes showed no central island, demonstrating the capability of the excimer laser calibration system for predicting the beam profile accurately and subsequently calibrating the system accordingly.

Mrochen et al. $^{2}$ performed a study on freshly enucleated bovine corneas and PMMA plates to evaluate the influence of the dynamics of the laser ablation process, including the pulse energies and radiant exposures. The shot pattern for the myopic correction was maintained during all experiments, and the pulse laser energy was adjusted to achieve equal ablation depths for all repetition rates. Pulse energy, radiant exposure, and pulse duration were monitored to determine the required laser parameter. The variations (standard deviation) of the profile measurements were $\pm 0.45 \mu \mathrm{m}$ or less for PMMA and $\pm 1.50 \mu \mathrm{m}$ or less for bovine corneas. Increasing the repetition rate from 100 to $1050 \mathrm{~Hz}$ required an increase in peak radiant exposure from 400 to $530 \mathrm{~mJ} / \mathrm{cm}^{2}$ to achieve equal ablation for the myopic correction. The required increase in the mean radiant exposure ranged from 190 to $260 \mathrm{~mJ} / \mathrm{cm}^{2}$. Higher-repetition-rate excimer lasers required increased radiant exposure.

\subsection{Wax Impressions}

Efforts to better understand the processes of corneal ablation are aided by means to precisely measure corneal tissue ablation rates. $^{3}$ Fisher and Hahn used a white-light interferometer in a study to measure the depth of argon fluoride laser ablation craters and wax impressions of the ablation craters. The technique was validated using PMMA and was then used to measure the ablation rate of bovine corneal tissue. The ablation rate was measured as $0.47 \mu \mathrm{m} /$ pulse from direct measurement of the PMMA ablation craters and as $0.48 \mu \mathrm{m} /$ pulse from measurement of the corresponding wax impressions. Wax impressions provided a valid representation of ablation craters.

\subsection{Filofocon $A$}

New experimental corneal models in plastic (such as Filofocon A, a contact lens material) have been proposed recently to overcome some of the limitations of the theoretical approaches, aiming at improving the predictability of corneal reshaping by laser ablation. ${ }^{4}$ Dorronsoro et al. obtained the effective absorption coefficient and the ablation thresholds of Folifocon A and PMMA as a function of the number of pulses. Both materials follow a Beer-Lambert law in the range of fluences used in refractive surgery, and the number of incubation pulses (that is quantified as the number of the first pulses that do not contribute to ablation depth) $)^{5}$ is $<4$ in PMMA and 2 in Filofocon A, above $140 \mathrm{~mJ} / \mathrm{cm}^{2}$. Above 40 pulses for Filofocon A and 70 pulses for PMMA, ablation threshold and effective absorption coefficients can be considered constant (ablation threshold $=90 \mathrm{~mJ} / \mathrm{cm}^{2}$ and effective absorption coefficients $=36,000 \mathrm{~cm}^{-1}$, for Filofocon $\mathrm{A}$, and ablation threshold $=67 \mathrm{~mJ} / \mathrm{cm}^{2}$ and effective absorption coefficients $=52,000 \mathrm{~cm}^{-1}$ for PMMA, respectively). It was concluded that the absence of ablation artifacts (central islands), a lower number of incubation pulses, a lower pulsenumber dependence of the ablation threshold, and a good correspondence between effective absorption coefficient and the absorption coefficient alpha estimated from spectroscopic measurements make Filofocon A a more appropriate material than PMMA for experimental models in refractive surgery and for calibration of clinical lasers.

In another study, Filofocon A was used to study the ablation profiles and the outcomes of three state-of-the-art refractive surgery excimer lasers provided with narrow-beam flying spot and optimized algorithms (Ladarvision 4000, Alcon; Technolas 217 Z100, Bausch and Lomb; Allegretto wave Eye-Q, Wavelight). ${ }^{5}$ The 3-D ablation patterns produced by myopic laser corrections $(-9,-6$, and -3 D) on flat and spherical surfaces of Filofocon A were measured using high-resolution optical profilometry. The artificial eyes used in this study consisted of plastic cylinders of Filofocon A, with a diameter of $12.7 \mathrm{~mm}$, finished either on a polished flat or spherical (7.8-mm nominal radii of curvature) surface. A comparison of the ablation patterns on flat and on spherical surfaces provided a measurement of the laser efficiency losses from the center to the periphery at each point of the spherical plastic corneas. This effect also varied across lasers, depending on their fluence (120 to $\left.400 \mathrm{~mJ} / \mathrm{cm}^{2}\right)$. The predicted postoperative corneal ablation shape, ablated volume, asphericity, and spherical aberration varied across lasers, as well as the relative contribution of ablation pattern designs and efficiency losses to the increased asphericity. Although the results showed that the algorithms have been optimized to reduce the induction of spherical aberration, they would still benefit from the application of correction factors for efficiency effects derived from a systematic approach using experimental plastic models.

\subsection{Poly (Ethylene Terephthalate)}

Poly (ethylene terephthalate) (PET) is a thermoplastic polymer, showing formation of a liquid layer during the laser ablation process. Rapid cooling of the surface after the laser application results in an amorphous surface layer, with lower molecular weight and lower density than the bulk polymer. Most literature studies deal with static ablation on calibration materials; however, Wagner ${ }^{6}$ studied the scanning ablation of PET at $193 \mathrm{~nm}$, using high fluence $\left(1 \mathrm{~J} / \mathrm{cm}^{2}\right)$. He studied the surface properties 
of the ablated microfluidic channels, comparing static ablation to scanning ablation with different parameters. The results showed that the properties of the ablated surfaces were modified, in terms of chemical properties and in terms of morphology, mainly due to the angle between the irradiated ramp forming under the beam and the nonirradiated surface, and the shape of the laser spot on the substrate. It was concluded that such morphological changes must be taken into account when fabricating devices, especially for which properties such as wettability, adhesion, and micro/nanoroughness are important. PET is also used in commercially available laser system as a calibration material. ${ }^{7}$

\section{Calibration Devices}

\subsection{Laser Profilometry}

The homogeneity of the ablation profile of excimer lasers can be disturbed by various reasons. Laser profilometry scans serve as a preoperative laser quality control if they can be correlated with clinical results. ${ }^{8}$ The laser profilometers estimate the ablation depth at each location on the ablation profile though the slope of laser light refracted from the surface location. The calibration process involves adjusting the laser systems to achieve a constant ablation depth per laser. Laser studies involving multiple laser platforms have indicated that there may be a correlation between results of laser surface profilometry scans and clinical outcomes, and surface profilometry may help to avoid undesirable clinical outcomes from ablation profile irregularities. ${ }^{9}$

\subsection{Power/Energy Meter}

Power/energy meter is the simplest method to control and calibrate a laser system. This holds the assumption that the spot size, shape, and pulse duration do not significantly change with time, hence, keeping the pulse energy constant means keeping the ablation rate constant. Following this principle, the WaveLight ${ }^{\circledR}$ EX500 Excimer laser features a high-resolution power/energy meter for an automated method for energy calibration, besides an ablation depth micrometer (which is used on a PMMA target), resulting in a claimed energy calibration within a tolerance range of $< \pm 1.5 \mu \mathrm{m}$ on a PTK treatment of 66.5- $\mu \mathrm{m}$ nominal depth. This means that the nominal energy per laser pulse is iteratively calibrated such that the ablation depth is reached between 65 and $68 \mu \mathrm{m}$ on PMMA.

\subsection{Shack-Hartmann Sensors}

The application of Shack-Hartmann sensors instigated the analysis of wavefront aberrations in the field of ophthalmology. ${ }^{9}$ Most commercial surgical excimer laser systems are calibrated by ablating plastic lenses, which are measured by lensometer and analyzed by a technician. The accuracy of this method is $\sim 0.25$ diopters (D) in sphere and cylinder power. Chernyak and Campbell ${ }^{10}$ measured an ablated plastic lens with a wavefront measurement device and compared the measured and analytically defined surface to validate the accuracy of a laser system. Somani et al. ${ }^{11}$ later developed a Shack-Hartmann sensor-based method to measure ablated plastic lenses for calibration and quality control of the excimer laser. Their tests revealed that the measurement of excimer-ablated plastic lenses agreed with high-precision surface profile scanner measurements within $0.10 \mathrm{D}$ and exceeding the repeatability by $0.01 \mathrm{D}$.

\subsection{White-Light Interferometers}

White-light interferometry is a noncontact optical method for surface height measurement on 3-D structures, often used as a calibration device in refractive surgery lasers. ${ }^{4,12}$ It makes use of the wave superposition principles to combine light waves in a way that their combination is used to extract information from instantaneous wavefronts. Hauge et al. ${ }^{13}$ examined ablated lenses by two Nidek EC-5000 scanning-slit excimer laser systems, using focimetry, interferometry, and mechanical surface profiling. In their results, interferometry revealed marked irregularity in the surface of negative corrections, which often had a positive "island" at their center.

\subsection{Laser Scanning Deflectometers}

Laser scanning deflectometers measure the pointwise deflection of a straight rays of light (originating from an array of points) through a refracting material, to estimate the refraction through backward ray tracing and line integration. Laser scanning deflectometers have been used to develop apparatuses for measuring the optical characteristic of an ablation standard object having a known ablation rate. Such apparatuses comprise a projecting optical system for projecting target luminous flux that is used in refractive power measurement. The luminous flux is projected onto a standard ablation object, and a detecting optical system is used for detecting the target luminous flux projected by the optical system. ${ }^{14,15}$ The obtained refraction is compared to the target refraction to calculate the calibration data.

\section{Methods to Calibrate the System}

A comparison of the different methods to calibrate the commercially available laser systems is presented in Table 1 . Different ablation profiles are employed by different laser systems available commercially, for example, Bausch and Lomb laser platforms use a single-point ablation profile, Wavelight and ZEiSS Meditec platforms use PTK-like ablation profiles, while SCHWIND, NIDEK, and Kera laser platforms rely on the refractive ablation profiles. Statistically, the standard error in a measurement process is inversely proportional to the square root of the number of measurements. Therefore, as a measure to reduce the error in calibration, multiple ablations and measurements are often designed in calibration tests.

\subsection{Refractive Power Versus Ablation Depth Versus Ablation Volume}

The goal of a laser-based refractive treatment is to make a refractive correction in the patient's eye. A defined ablation depth is targeted in the cornea, acting as a means to achieve the desired refractive change. For a single-point ablation, measuring the achieved ablation depth at a location makes for a technically sound method of calibration. However, for a multipoint ablation, several and varying ablation depths are achieved at different locations in the ablated profile. In such a case, the laser systems could be calibrated based on the maximum ablation depth at a particular location, average ablation depth of all the locations, or the average ablation depth within a region of interest on the ablated profile. Therefore, measuring the refractive change in the ablated material offers a validation of the aim of manipulating the refraction of the ablated material by a desired amount. It must also be noted that the conversion rates between the ablation depth and refractive power vary across materials and are highly 
Arba-Mosquera, Vinciguerra, and Verma: Review of technological advancements in calibration...

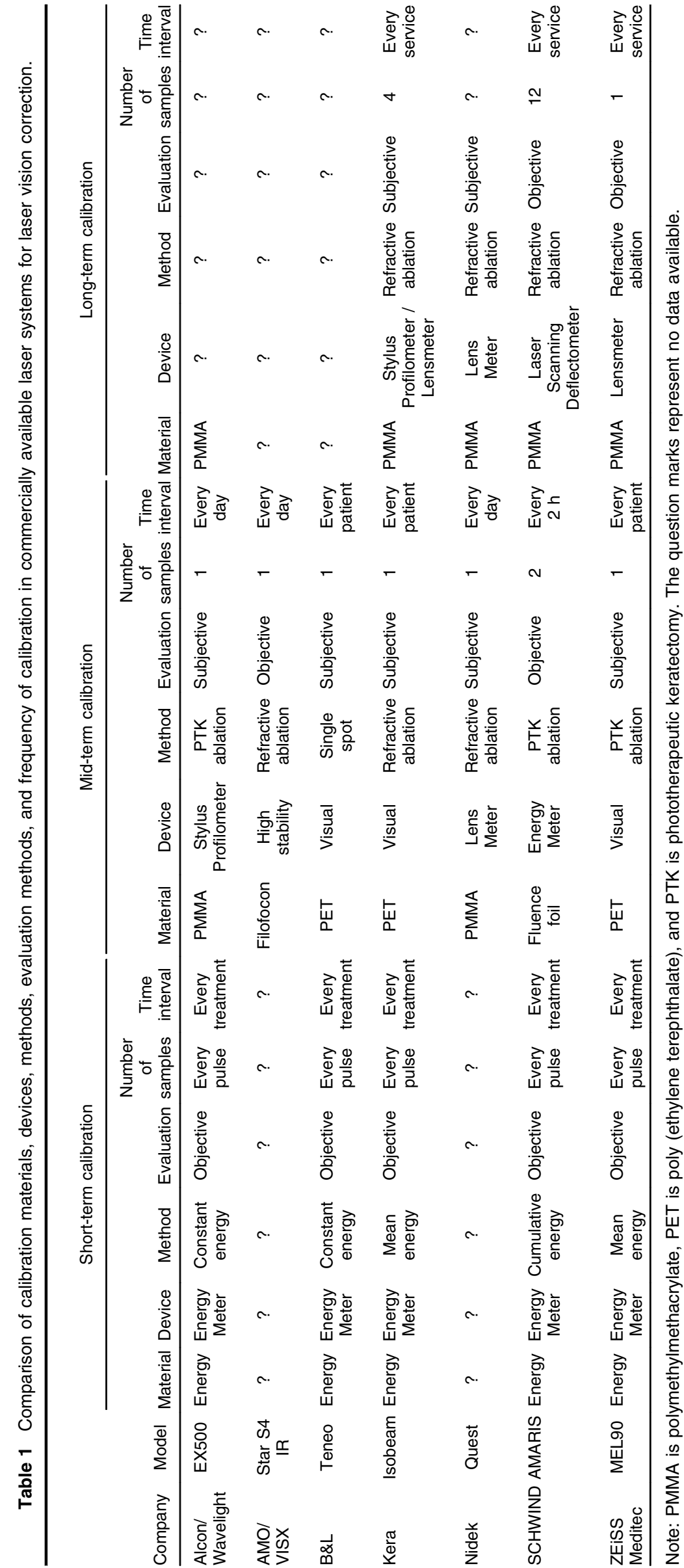


dependent on the surrounding conditions, substantiating the need for a customized complex modeling of the ablation process for an individualized condition. ${ }^{16}$

\subsection{Objective Versus Subjective Calibration}

The calibration data obtained from the calibration devices can be used to adjust the laser systems either objectively or subjectively (Fig. 1), where an objective calibration automatically adjusts the laser system based on the calibration data, and a subjective calibration involves a technician adjusting the system based on the subjective interpretation of the calibration measurements. Many commercially available systems, however, rely on a subjective adjustment of the laser systems performed by trained technicians (Table 1). The standards of technical training followed in the medical laser industry are certainly very high; however, subjective misinterpretation of the data might introduce some unwanted errors in the calibration process. On the other hand, objective calibrations need to incorporate plausibility tests and protection means against malfunctioning, since they do not have a human interface to validate how valuable the data are (e.g., bad spots in a Hartmann-Shack device).

\section{System Calibration and Ablation Roughness}

Plastic models, such as PMMA, are readily used to calibrate laser systems used in corneal tissue ablation. Hence, for a better calibration of the laser system, a higher fidelity is needed in measuring the ablated material. Surface roughness plays an important role in accurate measurement of ablation performance. Therefore, along with improvements in calibration systems, smoother ablations shall also be targeted for improving the fidelity and minimizing the measurement error during the calibration process.

Surface roughness after excimer laser ablation is a function of the amount of ablation. ${ }^{17}$ This has been previously determined using VISX 20/20 excimer laser ablations in PMMA. A linear increase was found in the surface roughness (increase of 25-nm roughness per micron of ablation) with the increase in refractive correction, measured using white-light interferometry microscope (Zygo Corp., Middlefield, Connecticut).

Surface roughness after ablation has been shown to vary in different excimer lasers systems. ${ }^{18}$ The spot positioning algorithm has a major influence on the resulting ablation smoothness, with the scanning excimer lasers based on flying spot technology rendering smoother ablations compared to older technologies. ${ }^{19}$ The flying spot technique was first proposed and patented by Lin, ${ }^{20,21}$ followed by their pioneering work regarding beam smoothness and ablation quality. In a study by Mrochen et al., ${ }^{22}$ bovine corneas and PMMA plates were photoablated using a $1050-\mathrm{Hz}$ excimer laser prototype for corneal laser surgery. Four temporal and spatial spot distributions (scan sequences) with different temporal overlapping factors were created for three myopic, three hyperopic, and three PTK ablation profiles. The temporal and spatial distributions of the laser spots (scan sequence) during a corneal laser procedure affected the surface quality and maximum ablation depth of the ablation profile. It was concluded that corneal laser surgery could theoretically benefit from smaller spot sizes and higher repetition rates. Levels of surface roughness produced by some older laser systems may be sufficient to degrade visual performance under some circumstances. This was shown by ablating a range of lenses of different powers in PMMA sheets using five disk operating system-based Nidek EC-5000 laser systems (Nidek Technologies, Gamagori, Japan) from different clinics. ${ }^{23}$ Surface quality was objectively assessed using profilometry. Average surface roughness was found to increase with lens power, roughness values being higher for negative lenses than for positive lenses. Losses in visual contrast sensitivity and acuity measured in two subjects were found to follow a similar pattern. Similar findings were shown in contrast sensitivity through ablated lenses affected by surface roughness in another study ${ }^{14}$ In a comparative study, Thomas et al. ${ }^{24}$ ablated the VISX calibration plastic and porcine cornea with standard -3.00-D, -6.00-D, and -9.00-D settings using four different excimer lasers. Electron microscopy and laser interferometry were used for qualitative evaluation of the ablated surfaces. Corneal ablation surface smoothness was graded by 10 independent observers. Calibration plastic ablated surfaces were evaluated quantitatively for smoothness by laser interferometry. In comparing ablation smoothness among various refractive powers $(-9.00 \mathrm{D},-6.00 \mathrm{D}$, and $-3.00 \mathrm{D})$, a trend was observed that indicated a correlation of higher refractive settings with decreasing surface smoothness. However, this trend was not statistically significant. The quantitative laser interferometry measurements supported the independent observer ranking of the autonomous flying small spot ablation profile as the smoothest. However, there were differences between the laser interferometry smoothness rankings and independent observer smoothness rankings.

\section{Other Considerations}

The current popularity of excimer laser refractive surgery suggests a need for continued research and refinements to further improve clinical outcomes. ${ }^{25}$ A fundamental limitation of current clinical systems is the lack of real-time feedback specifically addressing the laser-tissue interactions as directly related to laser ablation rates. A linear relationship has been observed between several cross-correlation metrics and the directly measured corneal ablation rate, yielding an average RMS predictive error of $3.9 \%$ using a 25 -shot average reflected waveform. Assessment of the cross-correlation approach for single-shot ablation data revealed a brief transient corresponding to the first few laser pulses, which is attributed to a slight hydration gradient near the surface of the de-epithelialized cornea.

\subsection{Cornea to Calibration Material Ratio}

The laser systems applied on the human cornea are calibrated using different calibration materials. Hence, the ratio of ablation efficiency between the human cornea and calibration material is very critical for a successful refractive procedure. ${ }^{26}$ This ratio highly depends on the laser beam characteristics. ArbaMosquera and Triefenbach proposed a model to convert the deviations in achieved ablation observed in PMMA to equivalent deviations in the cornea. They reported that radiant exposures from about 90 to about $500 \mathrm{~mJ} / \mathrm{cm}^{2}$ correspond to corneato-PMMA ablation ratios of about 9 and about 1.7 , respectively (about 7 and 1.3 optically). Simple Gaussian and flat-top laser beam profiles with a radiant exposure of $250 \mathrm{~mJ} / \mathrm{cm}^{2}$ corresponded to cornea-to-PMMA ablation ratios of $\sim 2.3$ and $\sim 1.6$, respectively (about 1.7 and about 1.2 optically). For a Gaussian beam of $160 \mathrm{~mJ} / \mathrm{cm}^{2}$ radiant exposure, a severe overcorrection of $+50 \%$ in PMMA corresponded only to an overcorrection of $+29 \%$ on corneal tissue, whereas a moderate overcorrection of $+20 \%$ in PMMA corresponded to an overcorrection of $+12 \%$ on corneal tissue. ${ }^{19}$ Another model from the same group 
introduced a method to convert the temperature rise during ablation observed in PMMA to equivalent temperature rises in the cornea. ${ }^{27}$

\subsection{Flat Calibration Versus Curved Ablation in Corneas}

There is a loss of ablation efficiency due to the corneal curvature and nonnormal incidence of the laser pulses at the edges of the ablation zone compared to the center of the ablation zone. ${ }^{28}$ This aspect is generally not considered while calibrating a laser system based on flat ablations performed on calibration materials. Dorronsoro et al. ${ }^{29}$ ablated flat and spherical PMMA surfaces with a standard refractive surgery laser system. They used the ratio of profiles on flat-to-spherical PMMA surfaces to estimate experimentally the radial change in ablation efficiency for PMMA and cornea. They reported that changes in ablation efficiency accounted for most of the asphericity increase found clinically, inducing spherical aberrations and by extension, other high-order aberrations, which result in a worsening of vision quality. They later invented a systematic method to obtain a correction factor for any ablation algorithm and laser system, which can be applied to prevent the induction of spherical aberrations. $^{30}$ Therefore, the calibration systems should be designed to also account for this strong change in ablation efficiency.

\subsection{Debris Removal in the Cornea Versus in the Calibration Material}

Air flow is a key factor in the evaluation of ablation patterns in refractive surgery using plastic models, as significant shielding effects are found with typical air-flow levels used under clinical conditions. This was shown in a study where an Allegretto EyeQ Laser platform (Wavelight $\mathrm{GmbH}$, Erlangen, Germany) was used to study the effect of air-flow speed on the ablation of artificial polymer corneas used for testing refractive surgery patterns. ${ }^{31}$ Flat samples of two materials (PMMA and Filofocon A) were ablated at four different air-flow conditions. The shape and profile of the ablated surfaces were measured with a precise noncontact optical surface profilometer. Significant asymmetries in the measured profiles were found when the ablation was performed with the clinical air aspiration system and also without air flow. Increasing air flow produced deeper ablations, improved symmetry, and increased the repeatability of the ablation pattern. Shielding of the laser pulse by the plume of smoke during the ablation of plastic samples reduced the central ablation depth by more than $40 \%$ with no-air flow, $30 \%$ with clinical air aspiration, and $5 \%$ with $1.15-\mathrm{m} / \mathrm{s}$ air flow. A simple model based on noninertial dragging of the particles by air flow predicted no central shielding with $2.3-\mathrm{m} / \mathrm{s}$ air flow and accurately predicted (within $2 \mu \mathrm{m}$ ) the decrease of central ablation depth by shielding. The shielding effects for PMMA and Filofocon A were similar despite the differences in the ablation properties of the materials and the different full-shielding transmission coefficient, which is related to the number of particles ejected and their associated optical behavior. The shielding effects could be avoided by tuning the air flow to the laser repetition rate. Similar findings in another study confirmed the need for more powerful debris removal systems and smart shot patterns in terms of thermal response to improve the performance of excimer lasers on PMMA. ${ }^{32}$

\subsection{Temperature Rise in the Cornea Versus in the Calibration Material}

The initial temperature of the ablated material also influences the measurement and subsequently the calibration of the laser systems. This was shown with an experimental argon fluoride excimer laser with a repetition rate of $1050 \mathrm{~Hz}$, a radiant exposure of $500 \mathrm{~mJ} / \mathrm{cm}^{2}$, and single pulse energy of $2.1 \mathrm{~mJ}$, which was used for photoablation of PMMA plates. ${ }^{33}$ The initial plate temperature varied from $10.1^{\circ} \mathrm{C}$ to $75.7^{\circ} \mathrm{C}$. The initial temperature was measured with an infrared camera, and the central ablation depth of a myopic ablation of $-9.00 \mathrm{D}$ with an optical zone of $6.5 \mathrm{~mm}$ was measured by means of a surface profiling system. The ablation depth increased linearly from 73.9 to $96.3 \mu \mathrm{m}$ within a temperature increase from $10.1^{\circ} \mathrm{C}$ to $75.7^{\circ} \mathrm{C}$ (increase rate of $0.32 \mu \mathrm{m} / \mathrm{K}$ ). The linear correlation was found to be significant $(P<0.05)$ with a coefficient of determination of $R^{2}=0.95$. Based on these results and assuming a standard room temperature of $20^{\circ} \mathrm{C}$, optimal plate temperature was calculated to be $15^{\circ} \mathrm{C}$ to $25^{\circ} \mathrm{C}$ to maintain an ablation within $0.25 \mathrm{D}$. Therefore, the temperature of PMMA plates for clinical laser calibration should be controlled ideally within a range of $\sim \pm 5^{\circ} \mathrm{C}$, to avoid visually significant refractive error due to calibration error.

\subsection{Short-Term Versus Long-Term Calibrations}

Most commercial laser systems used in refractive surgery follow different calibration steps at different time intervals. These can be broadly classified as short-, mid-, and long-term calibrations (Table 1).

Short-term calibration is performed in SCHWIND AMARIS, Wavelight, Nidek and ZEiSS Meditec MEL90 laser systems where every laser pulse delivered during the treatment is calibrated to a constant energy. This is followed by a cumulative energy control after every treatment in SCHWIND laser systems, energy control after every treatment in Wavelight and Nidek laser systems, and mean energy control after every treatment in MEL90 and Kera laser systems.

Mid-term calibration is done with a fluence test performed every $2 \mathrm{~h}$ in SCHWIND AMARIS and after every patient in ZEiSS Meditec MEL90 and Kera laser systems. Wavelight and Nidek laser systems use daily PMMA ablation tests for mid-term calibration of the systems.

Long-term calibration is performed with a PMMA ablation test in SCHWIND AMARIS, ZEiSS Meditec MEL90, and Kera laser systems at every technical service of the laser system.

\section{Conclusion}

Several groups have tested the accuracy of the developed calibration systems and methodologies. Surface roughness plays an important role in accurate measurement of ablation performance on calibration materials. Therefore, along with improvements in calibration systems, smoother ablations shall also be targeted for improving the fidelity and minimizing the measurement error during the calibration process. The ratio of ablation efficiency between the human cornea and calibration material is very critical and highly dependent on the laser beam characteristics and test conditions.

Defining proper test patterns for the routine check of ablative systems is not an easy task. There are many different components to be tested, and a single system test can only show a qualitative overall status of a system but, in general, cannot 
identify the specific sources of error when the PASS criteria are not met. For that reason, it is necessary to develop a comprehensive test for checking the overall status, including the spot roundness, scanner symmetry, scanning amplitude, scanner relative and absolute orientation, spot size and shape, calibration of centration, calibration of rotational orientation, and calibration of perpendicular orientation. Simple yet comprehensive set of patterns shall be designed, which are fast to perform and quick and easy to analyze and calibrate the overall status of a system. For the sake of conciseness, these aspects were not been included under the scope of this review article.

Few recommendations can be made here from the clinical standpoint. The calibration of the pulse energy is more critical than ablation depth as the energy can be technically manipulated with more accuracy, whereas calibration of ablation depth always depends on the calibration material and evaluation method and further involves conversion factors to estimate the performance on the human cornea. Due to the strong influence of environmental factors, such as the number of people in the operation room, temperature, humidity, dust, lighting etc., it is recommended to perform system calibration in conditions as close to the final environmental conditions seen in the operating room. PMMA is well established and readily used as a calibration material; however, researchers are also testing other potentially more suitable materials. Like any other machine involving very high accuracy for operation, the interval between two consecutive calibration routines for laser systems in laser vision correction, shall be as short as possible. However, the calibration routine shall not interfere with the normal operation of the system while operating on a patient. Beyond the calibration of the laser systems based on the numerical calibration factors evaluated either objectively or subjectively, special attention must be on qualitative evaluation of the ablated calibration material. For example, the inhomogeneity in the laser beam is one of the factors affecting roughness on the ablated material, which can be qualitatively analyzed using a high-resolution imaging modality. To ensure a complication-free procedure and faster recovery, the laser systems should be also optimized in this regard before application on human cornea.

There has been a steady progression in the accuracy of laser vision correction procedures, which has intern raised the patient expectations. Objective evaluation of the calibration data and corresponding adjustment of the laser systems at regular intervals is essential for the continuing success and further improvements in the outcomes of laser vision correction procedures.

\section{Disclosures}

The authors report no conflicts of interest and have no proprietary interest in any of the materials mentioned in this article. Shwetabh Verma and Samuel Arba-Mosquera are employees at SCHWIND Eye-Tech-Solutions, Kleinostheim, Germany.

\section{References}

1. J. D. Gottsch et al., "Excimer laser calibration system," J. Refractive Surg. 12(3), 401-411 (1996).

2. M. Mrochen et al., "Experimental setup to determine the pulse energies and radiant exposures for excimer lasers with repetition rates ranging from 100 to $1050 \mathrm{~Hz}$," J. Cataract Refractive Surg. 35(10), 18061814 (2009).

3. B. T. Fisher and D. W. Hahn, "Determination of excimer laser ablation rates of corneal tissue using wax impressions of ablation craters and white-light interferometry," Ophthalmic Surg. Lasers Imaging 35(1), 41-51 (2004).
4. C. Dorronsoro et al., "Suitability of Filofocon A and PMMA for experimental models in excimer laser ablation refractive surgery," Opt. Express 16(25), 20955-20967 (2008).

5. C. Dorronsoro et al., "Experimental evaluation of optimized ablation patterns for laser refractive surgery," Opt. Express 17(17), 1529215307 (2009).

6. F. R. Wagner, "Scanning excimer laser ablation of poly(ethylene terephthalate) (PET) and its application to rapid prototyping of channels for microfluidics," Thesis, EPFL, Lausanne (2000).

7. M. Dick and H. Vogelsang, "Method device and system for determining a system parameter of a laser beam treatment system," U.S. Patent No. 8303577 B2 (2012).

8. T. Anschutz and S. Pieger, "Correlation of laser profilometry scans with clinical results," J. Refractive Surg. 15(2), S252-S256 (1999).

9. B. C. Platt and R. Shack, "History and principles of Shack-Hartmann wavefront sensing," J. Refractive Surg. 17(5), S573-S577 (2001).

10. D. A. Chernyak and C. E. Campbell, "System for the design, manufacture, and testing of custom lenses with known amounts of high-order aberrations," J. Opt. Soc. Am. A. 20(11), 2016-2021 (2003).

11. S. Somani et al., "Shack-Hartmann-based wavefront analyzer for calibrating excimer lasers," J. Refractive Surg. 22(9), 938-942 (2006).

12. L. M. Shanyfelt, "ArF excimer laser corneal ablation: effects of laser repetition rate and fundamental laser-tissue coupling," $\mathrm{PhD}$ Dissertation, University of Florida (2008).

13. E. Hauge, S. A. Naroo, and W. N. Charman, "Poly(methyl methacrylate) model study of optical surface quality after excimer laser photorefractive keratectomy," J. Cataract Refractive Surg. 27(12), 20262035 (2001).

14. Y. Suzuki, "Apparatus for measuring optical characteristic, an apparatus for measuring an ablation rate comprising the same, and an apparatus comprising either the former or the latter," U.S. Patent No. 6245058 (B1) (2001).

15. S. Arba-Mosquera and N. Triefenbach, "Method for setting calibration data to control unit of laser ablation device, involves deriving new calibration data, and automatically storing new calibration data for control unit in laser ablation device," U.S. Patent No. DE102009016008 (A1) (2010).

16. S. Arba-Mosquera and N. Triefenbach, "Analysis of the cornea-toPMMA ablation efficiency rate," J. Mod. Opt. 59(10), 930-941 (2012).

17. J. Kemner, "Surface roughness in PMMA is linearly related to the amount of excimer laser ablation," J. Refractive Surg. 12(1), 171-174 (1996).

18. C. Argento et al., "Smoothness of ablation on acrylic by four different excimer lasers," J. Refractive Surg. 17(1), 43-45 (2001).

19. A. V. Doga, A. A. Shpak, and V. A. Sugrobov, "Smoothness of ablation on polymethylmethacrylate plates with four scanning excimer lasers," J. Refractive Surg. 20(5), S730-S733 (2004).

20. J. T. Lin, "Critical review on refractive surgical lasers," Opt. Eng. 34(3), 668-676 (1995).

21. J. T. Lin, "Multiwavelength solid state laser using frequency conversion techniques," U.S. Patent No. 5144630 A (1992)

22. M. Mrochen et al., "Influence of spatial and temporal spot distribution on the ocular surface quality and maximum ablation depth after photoablation with a $1050 \mathrm{~Hz}$ excimer laser system," J. Cataract Refractive Surg. 35(2), 363-373 (2009).

23. S. A. Naroo and W. N. Charman, "Surface roughness after excimer laser ablation using a PMMA model: profilometry and effects on vision," J. Refractive Surg. 21(3), 260-268 (2005).

24. J. W. Thomas et al., "Electron microscopy of surface smoothness of porcine corneas and acrylic plates with four brands of excimer laser," J. Refractive Surg. 19(6), 623-628 (2003).

25. B. T. Fisher and D. W. Hahn, "Real-time measurement of ArF excimer laser corneal tissue ablation rates using cross-correlation of laser waveforms," Opt. Express 19(5), 4231-4241 (2011).

26. J. H. Shen et al., "Ablation rate of PMMA and human cornea with a frequency-quintupled Nd:YAG laser (213 nm)," Lasers Surg. Med. 21(2), 179-185 (1997).

27. S. Arba-Mosquera and M. Shraiki, "Analysis of the PMMA and cornea temperature rise during excimer laser ablation," J. Mod. Opt. 57(5), 400-407 (2010).

28. S. Arba-Mosquera and D. de Ortueta, "Geometrical analysis of the loss of ablation efficiency at non-normal incidence," Opt. Express 16(6), 3877-3895 (2008). 
29. C. Dorronsoro et al., "Experiments on PMMA models to predict the impact of corneal refractive surgery on corneal shape," Opt. Express 14(13), 6142-6156 (2006).

30. S. Celestino, C. Diaz, and D. Reol, "Method of preventing the induction of aberrations in laser refractive surgery systems," U.S. Patent No. WO2005122873 (A1) (2005).

31. C. Dorronsoro et al., "Effect of air-flow on the evaluation of refractive surgery ablation patterns," Opt. Express 19(5), 4653-4666 (2011).

32. S. Arba-Mosquera and T. Klinner, "Improving the ablation efficiency of excimer laser systems with higher repetition rates through enhanced debris removal and optimized spot pattern," J. Cataract Refractive Surg. 40(3), 477-484 (2014).

33. J. Wernli et al., "Initial surface temperature of PMMA plates used for daily laser calibration affects the predictability of corneal refractive surgery," J. Refractive Surg. 28(9), 639-644 (2012).

Samuel Arba-Mosquera received his $\mathrm{PhD}$ in sciences of vision from the University of Valladolid in 2007 to 2012 and physics from the University of Santiago de Compostela in 1993 to 1998. He works as an optical/visual researcher at SCHWIND Eye-Tech-Solutions
(Germany), with 15 years of experience in R\&D in optics and vision and expertise in development of algorithms for refractive surgery.

Paolo Vinciguerra is the head of ophthalmology in Humanitas Research Hospital. He has an extensive surgical experience in cataract surgery performing over 5,000 interventions, over 2,000 transplantations, over 500 interventions with the excimer laser (refractive and therapeutic), more than 500 glaucoma interventions, and 2,000 retina interventions. He has been involved in the design and development of the excimer laser Nidek Co. Ltd. (since 1991) and holds international patents. Since 2005, he also has taught at Milan University.

Shwetabh Verma received his MSc degree in biomedical engineering from Heidelberg University, Germany, in 2012. He works as biomedical optics engineer at SCHWIND Eye-Tech-Solutions, Germany, with five years of experience in vision research and development of algorithms for refractive surgery. He is affiliated with Heidelberg University as a PhD candidate (medical physics). His recent published work and current research interests include optimizing ablation smoothness, perception of roughness in vision, and refractive surgery optimization algorithms. 